\title{
A linear, stabilized, non-spatial iterative, partitioned time stepping method for the nonlinear Navier-Stokes/Navier-Stokes interaction model
}

\author{
Jian $\mathrm{Li}^{1^{*}}$ (D, Pengzhan Huang ${ }^{2}$, Jian $\mathrm{Su}^{3}$ and Zhangxin Chen ${ }^{4}$
}

"Correspondence:

jiaaanli@gmail.com

'Department of Mathematics,

School of Arts and Sciences, Shaanxi

University of Science and

Technology, Xian, P.R. China

Full list of author information is

available at the end of the article

\begin{abstract}
In this paper, a linear, stabilized, non-spatial iterative, partitioned time stepping method is developed and studied for the nonlinear Navier-Stokes/Navier-Stokes interaction. A backward Euler scheme is utilized for the temporal discretization while a linear Oseen scheme for the trilinear term is used to affect the spatial discretization approximated by the equal order elements. Therefore, we only solve a linear Stokes problem without spatial iterative per time step for each individual domain. Then, the method exploits properties of the Navier-Stokes/Navier-Stokes system to establish the stability and convergence by rigorous analysis. Finally, numerical experiments are presented to show the performance of the proposed method.
\end{abstract}

MSC: $35 \mathrm{Q} 10 ; 65 \mathrm{~N} 30 ; 76 \mathrm{D} 05$

Keywords: Partitioned time stepping methods; Fluid-fluid interface; Navier-Stokes equations; Convergence; Numerical experiments

\section{Introduction}

The Navier-Stokes equations are useful because they describe the physics of many realistic problems of academic and industrial interest. They may be used to model weather, ocean currents, water flow, and many other phenomena. Many important applications need an accurate solution of multi-domain, multi-physics coupling of one fluid with another (e.g., the Navier-Stokes with the Navier-Stokes problems) [3, 4, 31, 32]. The uncoupled methods for two fluids are coupled through their shared interface by a rigid-lid coupling condition, i.e., no penetration and a slip with a friction condition allowing a jump in the tangential velocities across the shared interface [12]. Physics-based uncoupled methods are different from the traditional ones in the sense that they focus on decomposing different physical domains by directly using the given physical interface conditions, which is the key idea of the method proposed in this paper. Moreover, these methods allow existing highly optimized codes for each subproblem to be used in parallel as black boxes at each time step to solve the coupled problem.

Efficient stabilized finite element methods have been widely used in scientific computation to achieve high accuracy for the Navier-Stokes equations approximated by the equal

(c) The Author(s) 2019. This article is distributed under the terms of the Creative Commons Attribution 4.0 International License (http://creativecommons.org/licenses/by/4.0/), which permits unrestricted use, distribution, and reproduction in any medium, provided you give appropriate credit to the original author(s) and the source, provide a link to the Creative Commons license, and indicate if changes were made. 
order elements in practice. While these methods have been shown to be very successful, the theory ensuring their convergence and advantages for a coupled problem is still under development. Recently, some results have been obtained for partitioned time stepping methods for the fluid-fluid interaction by using the finite element methods [12, 13, 25]. In this paper, we shall follow the state-of-the-art convergence theory by using the geometric averaging at three time levels of the slip velocity at the interface to compute a friction coefficient and further establish stability and convergence of the presented method for the coupled fluid-fluid model. We stress that the extension of the general convergence theory to the partitioned time stepping method for the Navier-Stokes/Navier-Stokes interaction is derived from that in [12]. Here, in order to ensure the balance between the spatial and temporal computing allocation, an unconditional stable backward Euler scheme is utilized for the temporal discretization while the linear Oseen scheme is applied for the trilinear term with a non-spatial iterative correction per time step. The method presented results in a better coefficient matrix of the form $\left(a_{i j}\right)_{N \times N}=v\left(\nabla \phi_{i}, \nabla \phi_{j}\right)+\left((\mathbb{C} \cdot \nabla) \phi_{i}, \phi_{j}\right)$, improving the model presented with small viscosity $[16,17]$. However, the difficulty for the numerical analysis arises from the trilinear term and the whole system for the presented discrete finite element scheme.

The rest of paper is organized as follows. In Sect. 2, we introduce the fluid-fluid model using two Navier-Stokes problems. In Sect. 3, the stability of the Navier-Stokes/NavierStokes interaction model is analyzed. In Sect. 4, the convergence of the presented method is analyzed. Finally, we present several numerical examples to illustrate the features of the proposed method in Sect. 5.

\section{Preliminary}

A coupled Navier-Stokes/Navier-Stokes problem is stated as follows:

$$
\begin{aligned}
& u_{i, t}-v_{i} \Delta u_{i}+u_{i} \cdot \nabla u_{i}+\nabla p_{i}=f_{i} \quad \text { in } \Omega_{i}, \\
& -v_{i} n_{i} \cdot \nabla u_{i} \cdot \tau=k\left|u_{i}-u_{j}\right|\left(u_{i}-u_{j}\right) \cdot \tau \quad \text { on } I, \\
& u_{i} \cdot n_{i}=0 \quad \text { on } I, \\
& \nabla \cdot u_{i}=0 \quad \text { in } \Omega_{i}, \\
& u_{i}(x, 0)=u_{i}^{0}(x) \quad \text { in } \Omega_{i}, \\
& u_{i}=0 \quad \text { on } \Gamma_{i}=\partial \Omega_{i} \backslash I .
\end{aligned}
$$

Here, $i, j=1,2, i \neq j$. Let the domain $\Omega=\Omega_{1} \cup \Omega_{2}$ consist of two subdomains $\Omega_{1}$ and $\Omega_{2}$ of $R^{d}, d=2,3$, with the outward unit normal vectors $n_{1}$ and $n_{2}$, respectively, coupled across the interface $I$. The viscosity $v_{i}>0$, the body force $f_{i}:[0, T] \rightarrow H^{1}\left(\Omega_{i}\right)$, and the parameter $k \in R$ are given, $i=1$, 2. Also, $u_{i}: \Omega_{i} \times[0, T] \rightarrow R^{d}$ and $p_{i}: \Omega_{i} \times[0, T] \rightarrow R$ represent velocity and pressure on the subdomains $\Omega_{i}$, respectively, $i=1,2$.

For the mathematical problem (1)-(6), the following Hilbert spaces are introduced [1]:

$$
\begin{aligned}
& X_{i}=\left\{v_{i} \in\left[H^{1}\left(\Omega_{i}\right)\right]^{d}: v_{i}=0 \text { on } \Gamma_{i} \text { and } v_{i} \cdot n_{i}=0 \text { on } I\right\}, \\
& M_{i}=\left\{q_{i} \in L^{2}\left(\Omega_{i}\right): \int_{\Omega_{i}} q_{i} d x=0\right\} .
\end{aligned}
$$


Multiplying (1) by $v_{i} \in X_{i}$ and (4) by $q_{i} \in M_{i}$, integrating and applying the divergence theorem, the above coupled problem is equivalent to finding $\left(u_{i}, p_{i}\right) \in\left(X_{i}, M_{i}\right)$ such that

$$
\begin{aligned}
& \left(u_{i, t}, v_{i}\right)+a\left(u_{i}, v_{i}\right)-d\left(v_{i}, p_{i}\right)+b\left(u_{i}, u_{i}, v_{i}\right)+\kappa \int_{I}|[\mathbf{u}]|[\mathbf{u}] v_{i} d s=\left(f_{i}, v_{i}\right), \\
& d\left(u_{i}, q_{i}\right)=0, \quad \forall\left(v_{i}, q_{i}\right) \in\left(X_{i}, M_{i}\right),
\end{aligned}
$$

where $[\cdot]$ denotes the jump of the indicated quantity across the interface $I:[\mathbf{u}]=u_{i}-u_{j}$ and

$$
\left(u_{i, t}, v_{i}\right)=\int_{\Omega_{i}} \frac{\partial u_{i}}{\partial t} v_{i} d x, \quad i=1,2
$$

The continuous bilinear forms $a(\cdot, \cdot)$ and $d(\cdot, \cdot)$ are defined on $X_{i} \times X_{i}$ and $X_{i} \times M_{i}$, respectively, by

$$
\begin{aligned}
& a\left(u_{i}, v_{i}\right)=v_{i}\left(\nabla u_{i}, \nabla v_{i}\right), \quad u_{i}, v_{i} \in X_{i}, \\
& d\left(v_{i}, q_{i}\right)=-\left(v_{i}, \nabla q_{i}\right)=\left(\operatorname{div} v_{i}, q_{i}\right), \quad v_{i} \in X_{i}, q_{i} \in M_{i} .
\end{aligned}
$$

These bilinear terms satisfy the following continuity and inf-sup properties:

$$
\begin{aligned}
& \left|a\left(u_{i}, v_{i}\right)\right| \leq v\left\|\nabla u_{i}\right\|_{0}\left\|\nabla v_{i}\right\|_{0}, \\
& \left|d\left(v_{i}, p_{i}\right)\right| \leq C\left\|\nabla v_{i}\right\|_{0}\left\|p_{i}\right\|_{0}, \\
& \sup _{v_{i} \in X_{i}} \frac{\mid d\left(v_{i}, q_{i}\right)}{\left\|\nabla v_{i}\right\|_{0}} \geq \beta\left\|q_{i}\right\|_{0} \quad \forall q_{i} \in M_{i}, \beta>0,
\end{aligned}
$$

where the positive constants $C$ and $\beta$ only depend on $\Omega$. Similarly, by using the divergence theorem, (3) and (6), the trilinear term $b(\cdot, \cdot, \cdot)$ can be defined as follows [36]:

$$
\begin{aligned}
b\left(u_{i}, v_{i}, w_{i}\right) & =\frac{1}{2}\left(u_{i} \cdot \nabla v_{i}, w_{i}\right)+\frac{1}{2}\left(\left(\operatorname{div} u_{i}\right) v_{i}, w_{i}\right) \\
& =\frac{1}{2}\left(u_{i} \cdot \nabla v_{i}, w_{i}\right)-\frac{1}{2}\left(u_{i} \cdot \nabla w_{i}, v_{i}\right), \quad u_{i}, v_{i}, w_{i} \in X_{i} .
\end{aligned}
$$

Obviously, the trilinear term $b(\cdot, \cdot, \cdot)$ satisfies the following skew-symmetry property [36]:

$$
b\left(u_{i}, v_{i}, w_{i}\right)=-b\left(u_{i}, w_{i}, v_{i}\right) .
$$

A realistic model would contain many more complex terms. Here, we mainly focus on an algorithmic issue so we assume that the solution of (1)-(6) to be approximated is a strong solution. Moreover, the energetic stability of the monolithic problem is valid:

$$
\sum_{i=1}^{2}\left(\frac{1}{2} \frac{d}{d t}\left\|u_{i}\right\|_{0}^{2}+v_{i}\left\|\nabla u_{i}\right\|_{0}^{2}\right)+\kappa \int_{I}|[\mathbf{u}]|^{3} d s=\sum_{i=1}^{2}\left(f_{i}, v_{i}\right)
$$




\section{Stabilizations for Galerkin approximations}

Given a respective shape-regular and conforming triangulation $\mathcal{T}_{h_{i}}$ of $\Omega_{i}$, the finite element method is to solve (7) in two pairs of finite dimensional spaces $\left(X_{i}^{h}, M_{i}^{h}\right) \subset\left(X_{i}, M_{i}\right)$ $[9,11,15,36]$.

Stabilization of the Stokes' problem using local pressure projections dates back to the papers of Silvester [33, 34], Brecker and Braack [2], Brezzi and Fortin [6], Brezzi and Pitkiranta [7], Dohrmann and Bochev [14], Connors [23] and [8]. They provide a wide theoretical framework for these methods. The aim of the section is to give an elementary application in the spirit of these papers for a class of pressure projection method with equal order distribution for both velocity and pressure, which are computationally convenient and efficient in a parallel and multigrid context. Then, the unstable velocity-pressure pairs of the equal-order finite elements are defined as follows [22, 26-29, 38]:

$$
\begin{aligned}
& X_{i}^{h}=\left\{v_{h} \in X:\left.v_{h}\right|_{K} \in\left[R_{r}(K)\right]^{d}, \forall K \in K_{h}\right\}, \\
& M_{i}^{h}=\left\{q_{h} \in M:\left.q_{h}\right|_{K} \in R_{r}(K), \forall K \in K_{h}\right\}, \quad r=1,2 .
\end{aligned}
$$

In order to analyze the stabilzation of the Galerkin approximations for the NavierStokes/Navier-Stokes interaction, we assume that $\pi_{i}^{h}$ denotes the interpolation operator from the richer space $M_{h}$ into the smaller space $\bar{M}_{h} \subset M_{h}$ such that $X_{h} \times \bar{M}_{h}$ satisfies the inf-sup condition and $\operatorname{div} X_{h} \subset M_{h}$.

\section{Lemma 3.1 It holds that}

$$
\sup _{v_{i}^{h} \in X_{i}^{h}} \frac{d\left(v_{i}^{h}, q_{i}^{h}\right)}{\left\|\nabla v_{i}^{h}\right\|_{0}}+G^{1 / 2}\left(q_{i}^{h}, q_{i}^{h}\right) \geq \beta_{0}\left\|q_{i}^{h}\right\|_{0}, \quad q_{i}^{h} \in M_{i}^{h},
$$

where the positive constant $\beta_{0}$ only depends on $\Omega$ and the stabilized term $G(\cdot, \cdot)$ is defined as follows:

$$
G\left(q_{i}^{h}, q_{i}^{h}\right) \sim \begin{cases}\left\|q_{i}^{h}-\pi_{i} q_{i}^{h}\right\|_{0}, & r=1, \\ \left\|h \nabla\left(q_{i}^{h}-\pi_{i} q_{i}^{h}\right)\right\|_{0}, & r=2 .\end{cases}
$$

Proof For a bounded Lipschitz connected domain $\Omega$ and for any $p_{i}^{h} \in L^{2}(\Omega)$, there exist a positive constant $C_{0}>0$ and $v_{i} \in\left[H^{1}\left(\Omega_{i}\right)\right]^{d}$ satisfying

$$
\operatorname{div} v_{i}=p_{i}^{h}
$$

such that

$$
\left\|v_{i}\right\|_{1} \leq C_{0}\left\|p_{i}^{h}\right\|_{0}
$$

and

$$
\left\|p_{i}^{h}\right\|_{0}^{2}=d\left(v_{i}, p_{i}^{h}\right)
$$


Then, there exists a linear operator $\tilde{\pi}_{i}^{h}:\left[H^{1}\left(\Omega_{i}\right)\right]^{d} \rightarrow X_{h}$ such that the orthogonality relation holds $[5,10]$ :

$$
\begin{aligned}
& \left(v_{i}-\tilde{\pi}_{i}^{h} v_{i}, q_{h}\right)=0, \quad \forall q_{h} \in M_{h}, \\
& \left\|\nabla \tilde{\pi}_{i}^{h} v_{i}\right\|_{0} \leq C\left\|v_{i}\right\|_{1} \leq C_{1}\left\|p_{i}^{h}\right\|_{0},
\end{aligned}
$$

where $C_{1}$ only depends on $\Omega$. Noting that $\operatorname{div} \tilde{\pi}_{h} v \in M_{h}$ and thus $d\left(\tilde{\pi}_{h} v, p_{i}^{h}-\pi_{h} p_{i}^{h}\right)=0$, and using the definition of $\tilde{\pi}_{h}$, we obtain

$$
\begin{aligned}
\left\|p_{i}^{h}\right\|_{0}^{2} & =d\left(v_{i}, p_{i}^{h}\right) \\
& =d\left(v_{i}, p_{i}^{h}-\pi_{i}^{h} p_{i}^{h}\right)+d\left(v_{i}, \pi_{i}^{h} p_{i}^{h}\right) \\
& =d\left(v_{i}-\tilde{\pi}_{h} v_{i}, p_{i}^{h}-\pi_{i}^{h} p_{i}^{h}\right)+d\left(\tilde{\pi}_{h} v_{i}, \pi_{i}^{h} p_{i}^{h}\right) \\
& =d\left(v_{i}-\tilde{\pi}_{h} v_{i}, p_{i}^{h}-\pi_{i}^{h} p_{i}^{h}\right)+d\left(\tilde{\pi}_{i}^{h} v_{i}, p_{i}^{h}\right),
\end{aligned}
$$

where

$$
\begin{aligned}
d\left(v_{i}-\tilde{\pi}_{i}^{h} v_{i}, p_{i}^{h}-\pi_{i}^{h} p_{i}^{h}\right) & \leq C\left\|p_{i}^{h}-\pi_{i}^{h} p_{i}^{h}\right\|_{0}\left\|v_{i}\right\|_{1} \\
& \leq G^{1 / 2}\left(p_{i}^{h}, p_{i}^{h}\right)\left\|p_{i}^{h}\right\|_{0}, \quad r=1,
\end{aligned}
$$

and

$$
\begin{aligned}
d\left(v_{i}-\tilde{\pi}_{i}^{h} v_{i}, p_{i}^{h}-\pi_{i}^{h} p_{i}^{h}\right) & =-\left(\nabla\left(p_{i}^{h}-\pi_{i}^{h} p_{i}^{h}\right), v_{i}-\tilde{\pi}_{i}^{h} v_{i}\right) \\
& \leq C h\left\|\nabla\left(p_{i}^{h}-\pi_{i}^{h} p_{i}^{h}\right)\right\|_{0}\left\|v_{i}\right\|_{1} \\
& \leq G^{1 / 2}\left(p_{i}^{h}, p_{i}^{h}\right)\left\|p_{i}^{h}\right\|_{0}, \quad r=2 .
\end{aligned}
$$

Therefore,

$$
\begin{aligned}
\sup _{v_{i}^{h} \in X_{i}^{h}} \frac{d\left(v_{i}^{h}, q_{i}^{h}\right)}{\left\|\nabla v_{i}^{h}\right\|_{0}}+G^{1 / 2}\left(p_{i}^{h}, p_{i}^{h}\right) & \geq \frac{d\left(\tilde{\pi}_{i} v_{i}, q_{i}^{h}\right)}{\left\|\nabla \tilde{\pi}_{i} v_{i}\right\|_{0}}+G^{1 / 2}\left(p_{i}^{h}, p_{i}^{h}\right) \\
& \geq \beta_{0}\left\|p_{i}^{h}\right\|_{0}^{2} .
\end{aligned}
$$

For more details, the result related to the well-posedness of the Navier-Stokes/NavierStokes interaction can be found in [12, 13, 18, 20, 30, 37].

\section{Stability}

In this section, we are now in a position to state a discrete finite element scheme. We let $\left(u_{i}^{n}, p_{i}^{n}\right)=:\left(u_{i, h}^{n}, p_{i, h}^{n}\right), i=1,2$, denote a discrete approximation to $u_{i}\left(t^{n}\right)$, where the discrete time $t^{n}$ is calculated from the uniform time step size $\tau=T / N$ by $t^{n}=n \tau, n=0,1, \ldots, N$.

From the point of view of implementation, the method presented consists of several subroutines for solving the nonlinear fluid-fluid interaction. First, the first guess $u_{i}^{0}$ can be defined by (5). Then, we solve the Stokes equations approximated by the lower order finite element pairs to obtain the second initial datum $u_{i}^{1}$. Furthermore, the following solution 
$u_{i}^{n+1}, n=0,1,2, \ldots$, can be obtained by the following equations (16) and (17). For the numerical treatment of the time derivative term, we use the fully discrete backward Euler approximation. As for the partitioned scheme, we apply the Oseen scheme with a non-spatial iterative correction to simplify the trilinear term per time step and further obtain a better stiffness matrix. Especially, recalling the standard geometric averaging of the jump in [12, 13], we replace the term $u_{j}^{n+1}\left|u_{i}^{n+1}-u_{j}^{n+1}\right|$ by $\left|u_{i}^{n}-u_{j}^{n}\right| u_{i}^{n+1}$ and $u_{j}^{n}\left|u_{i}^{n}-u_{j}^{n}\right|^{1 / 2}\left|u_{i}^{n-1}-u_{j}^{n-1}\right|^{1 / 2}$ in order to decouple the fluid-fluid interaction, which is also a key idea to obtain the unconditionally stable partitioning.

The linear, stabilized, non-spatial iterative, partitioned time stepping method is defined as follows:

Step I. Find $\left(u_{i}^{1}, p_{i}^{1}\right) \in X_{i}^{h} \times M_{i}^{h}$ satisfying the following Stokes equations:

$$
a\left(u_{i}^{1}, v_{i}\right)-d\left(v_{i}, p_{i}^{1}\right)+d\left(u_{i}^{1}, q_{i}\right)+G\left(p_{i}^{1}, q_{i}\right)=\left(f, v_{i}\right) \quad \forall\left(v_{i}, q_{i}\right) \in X_{i}^{h} \times M_{i}^{h} .
$$

Moreover, set the iterative step $m=1,2, \ldots$, the error of two successive solutions

$$
e_{i}^{m}=\sqrt{\left(u_{i}^{m}-u_{i}^{m-1}\right)^{2}+\left(p_{i}^{m}-p_{i}^{m-1}\right)^{2}}<\varepsilon
$$

with a sufficiently small iterative tolerance $\varepsilon>0$.

Step II. Solve the Navier-Stokes/Navier-Stokes interaction: Given $\tau>0, f_{i} \in\left[H^{-1}\left(\Omega_{i}\right)\right]^{d}$ $(i=1,2)$, find $\left(u_{1}^{n+1}, p_{1}^{n+1}\right) \in X_{1}^{h} \times M_{1}^{h}$ such that

$$
\begin{aligned}
& \frac{\left(u_{1}^{n+1}-u_{1}^{n}, v_{1}\right)}{\tau}+a\left(u_{1}^{n+1}, v_{1}\right)-d\left(v_{1}, p_{1}^{n+1}\right)+d\left(u_{1}^{n+1}, q_{1}\right)+G\left(p_{1}^{n+1}, q_{1}\right) \\
& \quad+b_{1}\left(u_{1}^{n}, u_{1}^{n+1}, v_{1}\right)+k \int_{I}\left|\left[\mathbf{u}^{n}\right]\right| u_{1}^{n+1} v_{1} d s-k \int_{I}\left|\left[\mathbf{u}^{n}\right]\right|^{1 / 2}\left|\left[\mathbf{u}^{n-1}\right]\right|^{1 / 2} u_{2}^{n} \cdot v_{1} d s \\
& =\left(f_{1}\left(t^{n+1}\right), v_{1}\right), \quad \forall\left(v_{1}, q_{1}\right) \in X_{1}^{h} \times M_{1}^{h},
\end{aligned}
$$

and $\left(u_{2}^{n+1}, p_{2}^{n+1}\right) \in X_{2}^{h} \times M_{2}^{h}$ such that

$$
\begin{aligned}
& \frac{\left(u_{2}^{n+1}-u_{2}^{n}, v_{2}\right)}{\tau}+a\left(u_{2}^{n+1}, v_{2}\right)-d\left(v_{2}, p_{2}^{n+1}\right)+d\left(u_{2}^{n+1}, q_{2}\right)+G\left(p_{2}^{n+1}, q_{2}\right) \\
& \quad+b_{2}\left(u_{2}^{n}, u_{2}^{n+1}, v_{2}\right)+k \int_{I}\left|\left[\mathbf{u}^{n}\right]\right| u_{2}^{n+1} v_{2} d s-k \int_{I}\left|\left[\mathbf{u}^{n}\right]\right|^{1 / 2}\left|\left[\mathbf{u}^{n-1}\right]\right|^{1 / 2} u_{1}^{n} \cdot v_{2} d s \\
& =\left(f_{2}\left(t^{n+1}\right), v_{2}\right) \quad \forall\left(v_{2}, q_{2}\right) \in X_{2}^{h} \times M_{2}^{h} .
\end{aligned}
$$

This, of course, dictates that the overall structure of the linear, stablized, non-spatial iterative, partition time step method will be much the same as for a standard finite element method, as described in [12]. The key point of the presented method is to use a linear, non-spatial iterative, partitioned time stepping method for the nonlinear NavierStokes/Navier-Stokes interaction model.

\section{Routine:}

$\left[u_{h}^{0}, p_{h}^{0}\right]=\operatorname{Stokes}\left(\mathcal{T}_{h}, f\right)$;

for $i=0,1,2, \ldots, T / d t$;

while $\left(e_{m}>\varepsilon\right)$ do

$\left(u_{h}^{m}, p_{h}^{m}\right) \rightarrow\left(u_{h}^{m-1}, p_{h}^{m-1}\right)$ 
$\left[u_{h}^{m}, p_{h}^{m}\right]=\mathbf{L N S}\left(\mathcal{T}_{h}, u_{h}^{m-1}, f\right)$

end while

end for

In this section, we aim to establish a result concerning the unconditional stability of the scheme (16)-(17).

Lemma 4.1 Let $u_{i}^{n}, i=1,2, n=1,2, \ldots, m$, be the solutions of equations (16) and (17). Then we have the following energy inequality:

$$
\begin{aligned}
\| \mathbf{u}^{m+1} & \left\|_{0}^{2}+\sum_{n=1}^{m}\right\| \mathbf{u}^{n+1}-\mathbf{u}^{n} \|_{0}^{2}+\tau \sum_{n=1}^{m}\left(v_{1}\left\|\nabla u_{1}^{n+1}\right\|_{0}^{2}+v_{2}\left\|\nabla u_{2}^{n+1}\right\|_{0}^{2}\right) \\
& +\kappa \tau \int_{I}\left|\left[\mathbf{u}^{n}\right]\right|\left(\left|u_{1}^{n+1}\right|^{2}+\left|u_{2}^{n+1}\right|^{2}\right) d s \\
\leq & \left\|\mathbf{u}^{1}\right\|_{0}^{2}+\kappa \tau \int_{I}\left|\left[\mathbf{u}^{0}\right]\right|\left(\left|u_{1}^{1}\right|^{2}+\left|u_{2}^{1}\right|^{2}\right) d s \\
& +\sum_{n=1}^{m}\left(\frac{\tau}{v_{1}}\left\|f_{1}\left(t^{n+1}\right)\right\|_{0}^{2}+\frac{\tau}{v_{2}}\left\|f_{2}\left(t^{n+1}\right)\right\|_{-1}^{2}\right),
\end{aligned}
$$

where $u_{i}^{n}=\left(u_{1}^{n}, u_{2}^{n}\right)$ with the norm $\left\|\mathbf{u}^{n}\right\|_{0}=\left(\sum_{i=1}^{2}\left\|u_{i}^{n}\right\|_{0}^{2}\right)^{1 / 2}$.

Proof Noting that

$$
b\left(u_{i}^{n}, u_{i}^{n+1}, u_{i}^{n+1}\right)=0,
$$

we start by testing (16) and (17) with $\left(v_{i}, q_{i}\right)=2\left(\tau u_{i}^{n+1}, p_{i}^{n+1}\right)$, respectively, to obtain

$$
\begin{aligned}
& 2\left(u_{i}^{n+1}-u_{i}^{n}, u_{i}^{n+1}\right)+2 v_{i} \tau\left\|\nabla u_{i}^{n+1}\right\|_{0}^{2}+2 G\left(p_{i}^{n+1}, p_{i}^{n+1}\right) \\
& \quad+2 \kappa \tau\left(\int_{I}\left|u_{i}^{n+1}\right|^{2}\left|\left[\mathbf{u}^{n}\right]\right| d s-\int_{I}\left|\left[\mathbf{u}^{n}\right]\right|^{1 / 2}\left|\left[\mathbf{u}^{n-1}\right]\right|^{1 / 2} u_{i+1}^{n} \cdot u_{i}^{n+1} d s\right) \\
& =2 \tau\left(f_{i}\left(t^{n+1}\right), u_{i}^{n+1}\right) \quad \forall\left(v_{i}, q_{i}\right) \in X_{i}^{h} \times M_{i}^{h}, i=1,2,
\end{aligned}
$$

where $u_{3}=u_{1}$ when the index is out of bounds. Using the identity

$$
2(a-b, a)=a^{2}+(a-b)^{2}-b^{2},
$$

and combing with (19) with $i=1,2$, we obtain the following result:

$$
\begin{aligned}
& \left\|\mathbf{u}^{n+1}\right\|_{0}^{2}-\left\|\mathbf{u}^{n}\right\|_{0}^{2}+\left\|\mathbf{u}^{n+1}-\mathbf{u}^{n}\right\|_{0}^{2}+2 v_{1} \tau\left\|\nabla u_{1}^{n+1}\right\|_{0}^{2}+2 v_{2} \tau\left\|\nabla u_{2}^{n+1}\right\|_{0}^{2} \\
& \quad+2 \kappa \tau \sum_{i=1}^{2}\left(\int_{I}\left|u_{i}^{n+1}\right|^{2}\left|\left[\mathbf{u}^{n}\right]\right| d s-\int_{I}\left|\left[\mathbf{u}^{n}\right]\right|^{1 / 2}\left|\left[\mathbf{u}^{n-1}\right]\right|^{1 / 2} u_{i+1}^{n} \cdot u_{i}^{n+1} d s\right) \\
& \quad+2 G\left(p_{i}^{n+1}, p_{i}^{n+1}\right) \\
& =2 \tau \sum_{i=1}^{2}\left(f_{i}\left(t^{n+1}\right), u_{i}^{n+1}\right),
\end{aligned}
$$


where

$$
\begin{aligned}
& \kappa\left(\int_{I}\left|u_{i}^{n+1}\right|^{2}\left|\left[\mathbf{u}^{n}\right]\right| d s-\int_{I}\left|\left[\mathbf{u}^{n}\right]\right|^{1 / 2}\left|\left[\mathbf{u}^{n-1}\right]\right|^{1 / 2} u_{i+1}^{n} \cdot u_{i}^{n+1} d s\right) \\
& =\frac{\kappa}{2} \int_{I}\left|u_{i}^{n+1}\right|^{2}\left|\left[\mathbf{u}^{n}\right]\right| d s-\frac{\kappa}{2} \int_{I}\left|u_{i+1}^{n}\right|^{2}\left|\left[\mathbf{u}^{n-1}\right]\right| d s \\
& \quad+\left.\frac{\kappa}{2} \int_{I}\left|u_{i}^{n+1}\right|\left[\mathbf{u}^{n}\right]\right|^{1 / 2}-\left.u_{i+1}^{n}\left|\left[\mathbf{u}^{n-1}\right]\right|^{1 / 2}\right|^{2} d s, \\
& 2 \tau\left|\sum_{i=1}^{2}\left(f_{i}\left(t^{n+1}\right), u_{i}^{n+1}\right)\right| \\
& \leq 2 \tau \gamma \sum_{i=1}^{2}\left\|f_{i}\left(t^{n+1}\right)\right\|_{0}\left\|\nabla u_{i}^{n+1}\right\|_{0} \\
& \leq \sum_{i=1}^{2}\left(\tau v_{i}\left\|\nabla u_{i}^{n+1}\right\|_{0}^{2}+\frac{\tau \gamma^{2}}{v_{i}}\left\|f_{i}\left(t^{n+1}\right)\right\|_{0}^{2}\right),
\end{aligned}
$$

where the positive constant is derived from the Poincare inequality. Then, substituting these into (21), we infer that

$$
\begin{aligned}
& \left\|\mathbf{u}^{n+1}\right\|_{0}^{2}-\left\|\mathbf{u}^{n}\right\|_{0}^{2}+\left\|\mathbf{u}^{n+1}-\mathbf{u}^{n}\right\|_{0}^{2}+v_{1} \tau\left\|\nabla u_{1}^{n+1}\right\|_{0}^{2}+v_{2} \tau\left\|\nabla u_{2}^{n+1}\right\|_{0}^{2} \\
& \quad+\kappa \tau \int_{I}\left\{\left|u_{1}^{n+1}\right|^{2}+\left|u_{2}^{n+1}\right|^{2}\right\}\left|\left[\mathbf{u}^{n}\right]\right| d s-\kappa \tau \int_{I}\left\{\left|u_{1}^{n}\right|^{2}+\left|u_{2}^{n}\right|^{2}\right\}\left|\left[\mathbf{u}^{n-1}\right]\right| d s \\
& \quad+\left.\kappa \tau \sum_{i=1}^{2}\left|u_{i}^{n+1}\right|\left[\mathbf{u}^{n}\right]\right|^{1 / 2}-\left.u_{i+1}^{n}\left|\left[\mathbf{u}^{n-1}\right]\right|^{1 / 2}\right|^{2} d s \\
& \leq \tau \sum_{i=1}^{2} \frac{\left\|f_{i}\left(t^{n+1}\right)\right\|_{0}^{2}}{v_{i}} .
\end{aligned}
$$

Summing over $n=1,2, \ldots, m$ yields the desired result.

\section{Convergence}

In this section, we consider the convergence of the presented method for the NavierStokes/Navier-Stokes interaction. First, we provide the discrete Gronwall's inequality [21, 24], which will be useful in the subsequent analysis.

Let $a^{n+1}, b^{n+1}, c^{n+1}, d^{n+1}$ and $D^{n+1}, n=0,1,2, \ldots, m$, be five nonnegative sequences satisfying

$$
a^{m+1}+\sum_{n=0}^{m} b^{n+1}+\tau \sum_{n=0}^{m} c^{n+1} \leq C_{1}+C_{2} \tau \sum_{n=0}^{m} D^{n+1} a^{n+1}+C_{3} \tau \sum_{n=0}^{m} d^{n+1}
$$

Then we have the following result:

$$
a^{m+1}+\sum_{n=0}^{m} b^{n+1}+\tau \sum_{n=0}^{m} c^{n+1} \leq \exp \left(C_{2} \tau \sum_{n=0}^{m} \sigma^{n+1}\right)\left(C_{1}+C_{3} \tau \sum_{n=0}^{m} d^{n+1}\right)
$$


where

$$
\sigma^{n+1}=\frac{D^{n+1}}{1-\tau D^{n+1}}
$$

In order to analyze convergence of the partitioned time stepping methods for the fluid-fluid interaction, we introduce the following Stokes projection by finding $\left(R_{h}\left(v_{i}, q_{i}\right)\right.$, $\left.Q_{h}\left(v_{i}, q_{i}\right)\right) \in X_{i}^{h} \times M_{i}^{h}$ such that

$$
\begin{aligned}
& a\left(v_{i}-R_{h}\left(v_{i}, q_{i}\right), v_{h}\right)-d\left(v_{h}, q_{i}-Q_{h}\left(v_{i}, q_{i}\right)\right)+d\left(v_{i}-R_{h}\left(v_{i}, q_{i}\right), q_{h}\right)=0, \\
& \quad\left(v_{h}, q_{h}\right) \in X_{i}^{h} \times M_{i}^{h},
\end{aligned}
$$

which is well-defined and satisfies the following optimal approximation property:

$$
\begin{aligned}
& \left\|v_{i}-R_{h}\left(v_{i}, q_{i}\right)\right\|_{0}+h\left(\left\|\nabla\left(v_{i}-R_{h}\left(v_{i}, q_{i}\right)\right)\right\|_{0}+\left\|q_{i}-Q_{h}\left(v_{i}, q_{i}\right)\right\|_{0}\right) \\
& \quad \leq C h^{2}\left(\left\|v_{i}\right\|_{2}+\left\|q_{i}\right\|_{1}\right), \quad i=1,2 .
\end{aligned}
$$

Theorem 5.1 Assume that the initial data $u_{i}^{0}$ and $u_{i}^{1}$ satisfy the following estimate:

$$
\left\|\nabla\left(u_{i}\left(t^{0}\right)-u_{i}^{0}\right)\right\|_{0}+\left\|\nabla\left(u_{i}\left(t^{1}\right)-u_{i}^{1}\right)\right\|_{0} \leq C h, \quad i=1,2 .
$$

Moreover, the time step $\tau$ satisfies the relation $\tau<1 / D^{n+1}$ with $D^{n+1}$ defined by (43) below. Let $\left(u_{i}, p_{i}\right)$ and $\left(u_{i}^{n+1}, p_{i}^{n+1}\right)$ be the solutions of (1)-(6) and (16)-(17), respectively, with $u_{i} \in L^{2}\left([0, T], H^{2}\left(\Omega_{i}\right) \cap X_{i}\right), p_{i} \in L^{2}\left([0, T], H^{1}\left(\Omega_{i}\right) \cap M_{i}\right), u_{i, t} \in L^{2}\left([0, T], X_{i}\right)$ and $u_{i, t t} \in$ $L^{2}\left([0, T], L^{2}\left(\Omega_{i}\right)\right)$. Then it holds that

$$
\begin{aligned}
& \tau \sum_{n=0}^{m}\left(v_{1}\left\|\nabla\left(u_{1}\left(t^{n+1}\right)-u_{1}^{n+1}\right)\right\|_{0}^{2}+v_{2}\left\|\nabla\left(u_{2}\left(t^{n+1}\right)-u_{2}^{n+1}\right)\right\|_{0}^{2}\right) \\
& \quad \leq C\left(\tau^{2}+h^{2 r}\right), \quad r=1,2
\end{aligned}
$$

where $C$ denotes a positive constant depending on the data $\left(v_{i}, \Omega_{i}, u_{i}, p_{i}, f_{i}\right), i=1,2$, which may stand for different values at different occurrences.

Proof Here, we analyze convergence on each subdomain independently. For convenience, we set $\left(e_{i}^{j}, \eta_{i}^{j}\right)=\left(R_{h} u_{i}\left(t^{j}\right)-u_{i}^{j}, Q_{h} p_{i}\left(t^{j}\right)-p_{i}^{j}\right)$ and $E_{i}^{j}=u_{i}\left(t^{j}\right)-R_{h} u_{i}\left(t^{j}\right)$. First, using the Stokes projection, we subtract (16) or (17) from (7) with $\left(v_{i}, q_{i}\right)=\left(e_{i}^{n+1}, \eta_{i}^{n+1}\right) \in X_{i}^{h} \times M_{i}^{h}$ to obtain

$$
\begin{aligned}
& \left(\frac{\partial u_{i}\left(t^{n+1}\right)}{\partial t}-\frac{\left(u_{i}^{n+1}-u_{i}^{n}\right)}{\tau}, e_{i}^{n+1}\right)+a\left(e_{i}^{n+1}, e_{i}^{n+1}\right)+2 G\left(\eta_{i}^{n+1}, \eta_{i}^{n+1}\right) \\
& \quad+b\left(u_{i}\left(t^{n+1}\right), u_{i}\left(t^{n+1}\right), e_{i}^{n+1}\right)-b\left(u_{i}^{n}, u_{i}^{n+1}, e_{i}^{n+1}\right) \\
& \quad+\kappa\left(\int_{I} u_{i}\left(t^{n+1}\right)\left|\left[\mathbf{u}\left(t^{n+1}\right)\right]\right| \cdot e_{i}^{n+1} d s-\int_{I} u_{i}^{n+1}\left|\left[\mathbf{u}^{n}\right]\right| \cdot e_{i}^{n+1} d s\right) \\
& \quad+\kappa\left(\int_{I} u_{j}^{n}\left|\left[\mathbf{u}^{n}\right]\right|^{1 / 2}\left|\left[\mathbf{u}^{n-1}\right]\right|^{1 / 2} \cdot e_{i}^{n+1} d s-\int_{I} u_{j}\left(t^{n+1}\right)\left|\left[\mathbf{u}\left(t^{n+1}\right)\right]\right| \cdot e_{i}^{n+1} d s\right) \\
& =2 G\left(p_{i}\left(t^{j+1}\right), \eta_{i}\right),
\end{aligned}
$$


where $i=1, j=2$ or $i=2, j=1$. We analyze each term in the above equality. Note that

$$
\begin{aligned}
( & \left.\frac{\partial u_{i}\left(t^{n+1}\right)}{\partial t}-\frac{\left(u_{i}^{n+1}-u_{i}^{n}\right)}{\tau}, e_{i}^{n+1}\right) \\
= & \frac{1}{\tau}\left(\left(u_{i}\left(t^{n+1}\right)-u_{i}^{n+1}\right)-\left(u_{i}\left(t^{n}\right)-u_{i}^{n}\right), e_{i}^{n+1}\right)-\frac{1}{\tau}\left(u_{i}\left(t^{n+1}\right)-u_{i}\left(t^{n}\right), e_{i}^{n+1}\right) \\
& \quad+\left(\frac{\partial u_{i}\left(t^{n+1}\right)}{\partial t}, e_{i}^{n+1}\right) \\
= & \frac{1}{\tau}\left(E_{i}^{n+1}-E_{i}^{n}, e_{i}^{n+1}\right)+\frac{1}{\tau}\left(e_{i}^{n+1}-e_{i}^{n}, e_{i}^{n+1}\right)-\left(R H S_{i}^{n+1}, e_{i}^{n+1}\right),
\end{aligned}
$$

where $\left(R H S_{i}^{n+1}, v\right)=\left(\frac{u_{i}\left(t^{n+1}\right)-u_{i}\left(t^{n}\right)}{\tau}-\frac{\partial u_{i}\left(t^{n+1}\right)}{\partial t}, v_{i}\right), i=1,2$. Also, we see that

$$
\begin{aligned}
& \left|\left(E_{i}^{n+1}-E_{i}^{n}, e_{i}^{n+1}\right)\right| \leq \frac{1}{4 \varepsilon_{1} v_{i}}\left\|E_{i}^{n+1}-E_{i}^{n}\right\|_{-1}^{2}+\varepsilon_{1} v_{i}\left\|\nabla e_{i}^{n+1}\right\|_{0}^{2}, \\
& \left|\left(R H S_{i}, e_{i}^{n+1}\right)\right| \leq \frac{1}{4 \varepsilon_{2} v_{i}}\left\|R H S_{i}^{n+1}\right\|_{-1}^{2}+\varepsilon_{2} v_{i}\left\|\nabla e_{i}^{n+1}\right\|_{0}^{2},
\end{aligned}
$$

where $\varepsilon_{i}>0, i=1,2$. For the trilinear terms, it is easy to see that

$$
\begin{aligned}
& b\left(u_{i}\left(t^{n+1}\right), u_{i}\left(t^{n+1}\right), e_{i}^{n+1}\right)-b\left(u_{i}^{n}, u_{i}^{n+1}, e_{i}^{n+1}\right) \\
&= b\left(u_{i}\left(t^{n+1}\right)-u_{i}\left(t^{n}\right), u_{i}\left(t^{n+1}\right), e_{i}^{n+1}\right)+b\left(E_{i}^{n}, u_{i}\left(t^{n+1}\right), e_{i}^{n+1}\right) \\
& \quad+b\left(e_{i}^{n}, u_{i}\left(t^{n+1}\right), e_{i}^{n+1}\right)-b\left(u_{i}^{n}, u_{i}\left(t^{n+1}\right)-u_{i}^{n+1}, e_{i}^{n+1}\right) \\
&=I_{1}+I_{2}+I_{3}+I_{4} .
\end{aligned}
$$

To estimate these trilinear terms, using a classical result in [36], we see that

$$
\begin{aligned}
\left|I_{1}\right| & \leq C\left\|\nabla\left(u_{i}\left(t^{n+1}\right)-u_{i}\left(t^{n}\right)\right)\right\|_{0}\left\|\nabla u_{i}\left(t^{n+1}\right)\right\|_{0}\left\|\nabla e_{i}^{n+1}\right\|_{0} \\
& \leq \varepsilon_{3} v_{i}\left\|\nabla e_{i}^{n+1}\right\|_{0}^{2}+\frac{C}{4 \varepsilon_{3} v_{i}}\left\|\nabla u_{i}\left(t^{n+1}\right)\right\|_{0}^{2}\left\|\nabla\left(u_{i}\left(t^{n+1}\right)-u_{i}\left(t^{n}\right)\right)\right\|_{0}^{2} .
\end{aligned}
$$

Applying the Young inequality and the skew-symmetry of the trilinear term yields that

$$
\begin{aligned}
\left|I_{2}+I_{4}\right| & =\left|b\left(E_{i}^{n}, u_{i}\left(t^{n+1}\right), e_{i}^{n+1}\right)-b\left(u_{i}^{n}, E_{i}^{n+1}, e_{i}^{n+1}\right)\right| \\
& \leq\left(\left\|\nabla u_{i}^{n}\right\|_{0}+\left\|\nabla u_{i}\left(t^{n+1}\right)\right\|_{0}\right)\left(\left\|\nabla E_{i}^{n}\right\|_{0}+\left\|\nabla E_{i}^{n+1}\right\|_{0}\right)\left\|\nabla e_{i}^{n+1}\right\|_{0} \\
& \leq \varepsilon_{4} v_{i}\left\|\nabla e_{i}^{n+1}\right\|_{0}^{2}+\frac{c}{4 \varepsilon_{4} v_{i}}\left(\left\|\nabla u_{i}^{n}\right\|_{0}^{2}+\left\|\nabla u_{i}\left(t^{n+1}\right)\right\|_{0}^{2}\right)\left(\left\|E_{i}^{n}\right\|_{0}^{2}+\left\|E_{i}^{n+1}\right\|_{0}^{2}\right) .
\end{aligned}
$$

For the third term $I_{3}$, using the following inequality [20]:

$$
\|\phi\|_{L^{4}} \leq C\|\phi\|_{0}^{\frac{1}{2}-\frac{1}{4}(d-2)}\|\nabla \phi\|_{0}^{\frac{1}{2}+\frac{1}{4}(d-2)} \quad \forall \phi \in X_{i}, d=2,3,
$$


Li et al. Boundary Value Problems

(2019) 2019:115

Page 11 of 19

and applying the Young inequality and the Cauchy-Schwarz inequality, we have

$$
\begin{aligned}
\left|I_{3}\right| & \leq C\left\|e_{i}^{n}\right\|_{0}^{1 / 2}\left\|\nabla e_{i}^{n}\right\|_{0}^{1 / 2}\left\|\nabla u_{i}\left(t^{n+1}\right)\right\|_{0}\left\|\nabla e_{i}^{n+1}\right\|_{0} \\
& \leq \varepsilon_{5} v_{i}\left\|\nabla e_{i}^{n+1}\right\|_{0}^{2}+C\left\|e_{i}^{n}\right\|_{0}\left\|\nabla e_{i}^{n}\right\|_{0}\left\|\nabla u_{i}\left(t^{n+1}\right)\right\|_{0}^{2} \\
& \leq \varepsilon_{5} v_{i}\left\|\nabla e_{i}^{n+1}\right\|_{0}^{2}+\varepsilon_{6} v_{i}\left\|\nabla e_{i}^{n}\right\|_{0}^{2}+C\left\|e_{i}^{n}\right\|_{0}^{2}\left\|\nabla u_{i}\left(t^{n+1}\right)\right\|_{0}^{4}
\end{aligned}
$$

for $d=2$, and

$$
\begin{aligned}
\left|I_{3}\right| & \leq C\left\|e_{i}^{n}\right\|_{0}^{1 / 4}\left\|\nabla e_{i}^{n}\right\|_{0}^{3 / 4}\left\|\nabla u_{i}\left(t^{n+1}\right)\right\|_{0}\left\|\nabla e_{i}^{n+1}\right\|_{0} \\
& \leq \varepsilon_{5} v_{i}\left\|\nabla e_{i}^{n+1}\right\|_{0}^{2}+C\left\|e_{i}^{n}\right\|_{0}^{1 / 2}\left\|\nabla e_{i}^{n}\right\|_{0}^{3 / 2}\left\|\nabla u_{i}\left(t^{n+1}\right)\right\|_{0}^{2} \\
& \leq \varepsilon_{5} v_{i}\left\|\nabla e_{i}^{n+1}\right\|_{0}^{2}+\varepsilon_{6} v_{i}\left\|\nabla e_{i}^{n}\right\|_{0}^{2}+C\left\|e_{i}^{n}\right\|_{0}^{2}\left\|\nabla u_{i}\left(t^{n+1}\right)\right\|_{0}^{8}
\end{aligned}
$$

for $d=3$.

Setting

$$
\begin{aligned}
& \overline{\left|\left[\mathbf{u}\left(t^{n+1}\right)\right]\right|}=\frac{1}{2}\left(\left|\left[\mathbf{u}\left(t^{n+1}\right)\right]\right|+\left|\left[\mathbf{u}\left(t^{n}\right)\right]\right|\right), \\
& \overline{\left|\mathbf{u}^{n}\right|}=\frac{1}{2}\left(\left|\mathbf{u}^{n}\right|+\left|\mathbf{u}^{n-1}\right|\right),
\end{aligned}
$$

and using the same approach as in [12], we get

$$
\begin{aligned}
I_{5}= & \int_{I} u_{i}\left(t^{n+1}\right)\left|\left[\mathbf{u}\left(t^{n+1}\right)\right]\right| \cdot e_{i}^{n+1} d s-\int_{I} u_{i}^{n+1}\left|\left[\mathbf{u}^{n}\right]\right| \cdot e_{i}^{n+1} d s \\
= & \int_{I} u_{i}\left(t^{n+1}\right)\left(\left|\left[\mathbf{u}\left(t^{n+1}\right)\right]\right|-\left|\left[\mathbf{u}\left(t^{n}\right)\right]\right|\right) \cdot e_{i}^{n+1} d s \\
& +\int_{I} u_{i}\left(t^{n+1}\right)\left(\left|\left[\mathbf{u}\left(t^{n}\right)\right]\right|-\left|\left[P_{h} \mathbf{u}\left(t^{n}\right)\right]\right|\right) \cdot e_{i}^{n+1} d s \\
& +\int_{I} u_{i}\left(t^{n+1}\right)\left(\left|\left[P_{h} \mathbf{u}\left(t^{n}\right)\right]\right|-\left|\left[\mathbf{u}^{n}\right]\right|\right) \cdot e_{i}^{n+1} d s \\
& +\int_{I} E_{i}^{n+1}\left|\left[\mathbf{u}^{n}\right]\right| \cdot e_{i}^{n+1} d s+\int_{I}\left|\left[\mathbf{u}^{n}\right]\right|\left|e_{i}^{n+1}\right|^{2} d s \\
\leq & \int_{I} u_{i}\left(t^{n+1}\right)\left(\left|\left[\mathbf{u}\left(t^{n+1}\right)\right]\right|-\left|\left[\mathbf{u}\left(t^{n}\right)\right]\right|\right) \cdot e_{i}^{n+1} d s+\int_{I} u_{i}\left(t^{n+1}\right)\left|\left[E_{i}^{n}\right]\right| \cdot e_{i}^{n+1} d s \\
& +\int_{I} u_{i}\left(t^{n+1}\right)\left|\left[e_{i}^{n}\right]\right| \cdot e_{i}^{n+1} d s \\
& +\int_{I} E_{i}^{n+1}\left|\left[\mathbf{u}^{n}\right]\right| \cdot e_{i}^{n+1} d s+\int_{I}\left|\left[\mathbf{u}^{n}\right]\right|\left|e_{i}^{n+1}\right|^{2} d s .
\end{aligned}
$$

Noting that

$$
\begin{aligned}
& \left|\overline{\left|\left[\mathbf{u}\left(t^{n}\right)\right]\right|}-\right|\left[\mathbf{u}\left(t^{n+1}\right)\right]|| \\
& \quad=\frac{1}{2}\left(\left|\left[\mathbf{u}\left(t^{n}\right)\right]\right|-\left|\left[\mathbf{u}\left(t^{n+1}\right)\right]\right|\right)+\frac{1}{2}\left(\left|\left[\mathbf{u}\left(t^{n-1}\right)\right]\right|-\left|\left[\mathbf{u}\left(t^{n+1}\right)\right]\right|\right) \\
& \quad \leq \frac{1}{2}\left(\left|\left[\mathbf{u}\left(t^{n}\right)-\mathbf{u}\left(t^{n+1}\right)\right]\right|+\left|\left[\mathbf{u}\left(t^{n-1}\right)-\mathbf{u}\left(t^{n+1}\right)\right]\right|\right),
\end{aligned}
$$


Li et al. Boundary Value Problems

(2019) 2019:115

Page 12 of 19

and

$$
\begin{aligned}
& \left|\left[\mathbf{u}^{n}\right]\right|^{1 / 2}\left|\left[\mathbf{u}^{n-1}\right]\right|^{1 / 2}-\overline{\left|\left[\mathbf{u}^{n}\right]\right|} \mid \\
& \quad=\frac{1}{2}\left(\left|\left[\mathbf{u}^{n}\right]\right|^{1 / 2}-\left|\left[\mathbf{u}^{n-1}\right]\right|^{1 / 2}\right)^{2} \\
& \quad \leq \frac{1}{2}||\left[\mathbf{u}^{n}\right]|-|\left[\mathbf{u}^{n-1}\right]|| \\
& \quad \leq \frac{1}{2}\left|\left[\mathbf{u}^{n}-\mathbf{u}^{n-1}\right]\right| \\
& \quad \leq \frac{1}{2}\left|\left[\mathbf{u}^{n}-\mathbf{u}\left(t^{n}\right)+\mathbf{u}\left(t^{n-1}\right)-\mathbf{u}^{n-1}\right]\right|+\frac{1}{2}\left|\left[\mathbf{u}\left(t^{n}\right)-\mathbf{u}\left(t^{n-1}\right)\right]\right| \\
& \quad \leq\left|\overline{\left[\mathbf{e}^{n}\right]}\right|+\left|\overline{\left[\mathbf{E}^{n}\right]}\right|+\left|\overline{\left[\mathbf{e}^{n-1}\right]}+\overline{\left[\mathbf{E}^{n-1}\right]}\right|+\frac{1}{2}\left|\left[\mathbf{u}\left(t^{n}\right)-\mathbf{u}\left(t^{n-1}\right)\right]\right|,
\end{aligned}
$$

the following error bound holds:

$$
\begin{aligned}
I_{6}= & \int_{I} u_{j}^{n}\left|\left[\mathbf{u}^{n}\right]\right|^{1 / 2}\left|\left[\mathbf{u}^{n-1}\right]\right|^{1 / 2} \cdot e_{i}^{n+1} d s-\int_{I} u_{j}\left(t^{n+1}\right)\left|\left[\mathbf{u}\left(t^{n+1}\right)\right]\right| \cdot e_{i}^{n+1} d s \\
\leq & \int_{I} u_{j}^{n}\left(\left|\left[\mathbf{u}^{n}\right]\right|^{1 / 2}\left|\left[\mathbf{u}^{n-1}\right]\right|^{1 / 2}-\overline{\mathbf{u}^{n} \mid}\right) \cdot e_{i}^{n+1} d s+\int_{I} u_{j}^{n}\left(\overline{\left|\mathbf{u}^{n}\right|}-P_{h} \overline{\left|\mathbf{u}^{n}\right|}\right) \cdot e_{i}^{n+1} d s \\
& +\int_{I} u_{j}^{n}\left(P_{h} \overline{\left|\mathbf{u}^{n}\right|}-\left|\left[\mathbf{u}\left(t^{n}\right)\right]\right|\right) \cdot e_{i}^{n+1} d s \\
& +\int_{I} u_{j}^{n}\left(\overline{\left|\left[\mathbf{u}\left(t^{n+1}\right)\right]\right|}-\left|\left[\mathbf{u}\left(t^{n+1}\right)\right]\right|\right) \cdot e_{i}^{n+1} d s \\
& -\int_{I}\left(E_{j}^{n}+e_{j}^{n}\right)\left|\left[\mathbf{u}\left(t^{n+1}\right)\right]\right| \cdot e_{i}^{n+1} d s \\
& +\int_{I}\left(u_{j}\left(t^{n}\right)-u_{j}\left(t^{n+1}\right)\right)\left|\left[\mathbf{u}\left(t^{n+1}\right)\right]\right| \cdot e_{i}^{n+1} d s \\
\leq & \left.\int_{I} u_{j}^{n}\left|\left[\mathbf{u}\left(t^{n}\right)-\mathbf{u}\left(t^{n-1}\right)\right]\right| \cdot e_{i}^{n+1} d s+\int_{I} u_{j}^{n} \overline{\left|\left[\mathbf{E}^{n}\right]\right|} \cdot e_{i}^{n+1} d s+\int_{I} u_{j}^{n} \mid \overline{\left[\mathbf{e}^{n}\right.}\right] \mid \cdot e_{i}^{n+1} d s \\
& +\int_{I} u_{j}^{n}\left(\left|\left[\mathbf{u}\left(t^{n}\right)-\mathbf{u}\left(t^{n+1}\right)\right]\right|+\left|\left[\mathbf{u}\left(t^{n-1}\right)-\mathbf{u}\left(t^{n+1}\right)\right]\right|\right) \cdot e_{i}^{n+1} d s \\
& +\int_{I}\left(u_{j}\left(t^{n}\right)-u_{j}\left(t^{n+1}\right)\right)\left|\left[\mathbf{u}\left(t^{n+1}\right)\right]\right| \cdot e_{i}^{n+1} d s \\
& -\int_{I}\left(E_{j}^{n}+e_{j}^{n}\right)\left|\left[\mathbf{u}\left(t^{n+1}\right)\right]\right| \cdot e_{i}^{n+1} d s .
\end{aligned}
$$

Applying the same approach as in [12], we obtain

$$
\begin{aligned}
& \left|I_{5}+I_{6}\right| \\
& \leq \varepsilon_{7} v_{i}\left\|\nabla e_{i}^{n+1}\right\|_{0}^{2}+C v_{i}^{-3}\left(1+\left\|u_{2}^{n}\right\|_{I}^{4}+\left\|\left[\mathbf{u}\left(t^{n+1}\right)\right]\right\|_{I}^{4}\right)\left\|e_{i}^{n+1}\right\|_{0}^{2} \\
& \quad+\varepsilon_{8} \sum_{i=1}^{2} v_{i}\left(\left\|\nabla e_{i}^{n}\right\|_{0}^{2}+\left\|\nabla e_{i}^{n-1}\right\|_{0}^{2}\right)+C L^{n+1} P^{n+1} \\
& \quad+C M^{n+1} \sum_{i=1}^{2} v_{i}^{-3}\left(\left\|e_{i}^{n}\right\|_{0}^{2}+\left\|e_{i}^{n-1}\right\|_{0}^{2}\right),
\end{aligned}
$$


where $L^{n+1}, M^{n+1}$ and $P^{n+1}$ can be defined by the following bound terms on interface $I$ as follows:

$$
\begin{aligned}
L^{n+1}= & \sum_{i=1}^{2}\left(\left\|u_{i}\left(t^{n+1}\right)\right\|_{I}^{2}+\left\|u_{i}^{n+1}\right\|_{I}^{2}+\left\|u_{i}^{n}\right\|_{I}^{2}\right), \\
M^{n+1}= & \sum_{i=1}^{2}\left(\left\|u_{i}\left(t^{n+1}\right)\right\|_{I}^{4}+\left\|u_{i}^{n}\right\|_{I}^{4}\right), \\
P^{n+1}= & \sum_{i=1}^{2}\left\{\left(\left\|u_{i}\left(t^{n+1}\right)-u_{i}\left(t^{n}\right)\right\|_{I}^{2}+\left\|u_{i}\left(t^{n+1}\right)-u_{i}\left(t^{n-1}\right)\right\|_{I}^{2}\right)\right. \\
& \left.+\left(\left\|E_{i}^{n-1}\right\|_{I}^{2}+\left\|E_{i}^{n}\right\|_{I}^{2}+\left\|E_{i}^{n+1}\right\|_{I}^{2}\right)\right\} .
\end{aligned}
$$

Obviously, $\|\cdot\|_{I}$ is bounded by the corresponding $L^{2}$-norm. In addition, we can infer that the estimate of $P^{n+1}$ has the order of $O\left(\tau^{2}+h^{2 r}\right), r=1,2$.

Choosing $\varepsilon_{1}+\varepsilon_{2}+\varepsilon_{3}+\varepsilon_{4}+\varepsilon_{5}+\varepsilon_{7}=1 / 4, \varepsilon_{6}=1 / 8$, and $\varepsilon_{8}=1 / 16$, and combining all these inequalities with (30) yields that

$$
\begin{aligned}
& \frac{1}{2 \tau}\left(\left\|e_{i}^{n+1}\right\|_{0}^{2}-\left\|e_{i}^{n}\right\|_{0}^{2}+\left\|e_{i}^{n+1}-e_{i}^{n}\right\|_{0}^{2}\right)+\frac{3 v_{i}}{4}\left\|\nabla e_{i}^{n+1}\right\|_{0}^{2} \\
& \leq \\
& \quad C\left(\frac{1}{\tau}\left\|E_{i}^{n+1}-E_{i}^{n}\right\|_{-1}^{2}+\left\|R H S_{i}^{n+1}\right\|_{-1}^{2}+\left\|p_{i}-\Pi_{h} p_{i}\right\|_{0}^{2}\right) \\
& \quad+C\left\|\nabla u_{i}\left(t^{n+1}\right)\right\|_{0}^{2}\left\|\nabla\left(u_{i}\left(t^{n+1}\right)-u_{i}\left(t^{n}\right)\right)\right\|_{0}^{2} \\
& \quad+\left(\left\|\nabla u_{i}^{n}\right\|_{0}^{2}+\left\|\nabla u_{i}\left(t^{n+1}\right)\right\|_{0}^{2}\right)\left(\left\|E_{i}^{n}\right\|_{0}^{2}+\left\|E_{i}^{n+1}\right\|_{0}^{2}\right)+C\left\|e_{i}^{n}\right\|_{0}^{2}\left\|\nabla u_{i}\left(t^{n+1}\right)\right\|_{0}^{2^{d}} \\
& \quad+C v_{i}^{-3}\left(1+\left\|u_{2}^{n}\right\|_{I}^{4}+\left\|\left[\mathbf{u}\left(t^{n+1}\right)\right]\right\|_{I}^{4}\right)\left\|e_{i}^{n+1}\right\|_{0}^{2}+C L^{n+1} P^{n+1} \\
& \quad+\sum_{i=1}^{2} \frac{v_{i}}{16}\left(\left\|\nabla e_{i}^{n}\right\|_{0}^{2}+\left\|\nabla e_{i}^{n-1}\right\|_{0}^{2}\right)+\frac{v_{i}}{8}\left\|\nabla e_{i}^{n}\right\|_{0}^{2} \\
& \quad+C M^{n+1} \sum_{i=1}^{2} v_{i}^{-3}\left(\left\|e_{i}^{n}\right\|_{0}^{2}+\left\|e_{i}^{n-1}\right\|_{0}^{2}\right) .
\end{aligned}
$$

Summing over $i=1,2$ for the above inequality and moving the term on the 5 th line on the right-hand side of (40) to its left-hand side, t the term $\frac{v_{i}}{8}\left\|\nabla e_{i}^{n}\right\|_{0}^{2}$ can be exactly absorbed. Thus we find that

$$
\begin{aligned}
& \frac{1}{2 \tau}\left(\left\|\mathbf{e}^{n+1}\right\|_{0}^{2}-\left\|\mathbf{e}^{n}\right\|_{0}^{2}+\left\|\mathbf{e}^{n+1}-\mathbf{e}^{n}\right\|_{0}^{2}\right)+\sum_{i=1}^{2} \frac{v_{i}}{2}\left\|\nabla e_{i}^{n+1}\right\|_{0}^{2} \\
& \quad+\sum_{i=1}^{2} \frac{v_{i}}{4}\left(\left\|\nabla e_{i}^{n+1}\right\|_{0}^{2}-\left\|\nabla e_{i}^{n}\right\|_{0}^{2}\right)+\sum_{i=1}^{2} \frac{v_{i}}{16}\left(\left\|\nabla e_{i}^{n}\right\|_{0}^{2}-\left\|\nabla e_{i}^{n-1}\right\|_{0}^{2}\right) \\
& \leq C \sum_{i=1}^{2}\left(\frac{1}{\tau}\left\|E_{i}^{n+1}-E_{i}^{n}\right\|_{-1}^{2}+\left\|R H S_{i}^{n+1}\right\|_{-1}^{2}+\left\|p_{i}-\Pi_{h} p_{i}\right\|_{0}^{2}\right) \\
& \quad+C \sum_{i=1}^{2}\left\|\nabla u_{i}\left(t^{n+1}\right)\right\|_{0}^{2}\left\|\nabla\left(u_{i}\left(t^{n+1}\right)-u_{i}\left(t^{n}\right)\right)\right\|_{0}^{2}
\end{aligned}
$$




$$
\begin{aligned}
& +C \sum_{i=1}^{2}\left(\left\|\nabla u_{i}^{n}\right\|_{0}^{2}+\left\|\nabla u_{i}\left(t^{n+1}\right)\right\|_{0}^{2}\right)\left(\left\|E_{i}^{n}\right\|_{0}^{2}+\left\|E_{i}^{n+1}\right\|_{0}^{2}\right)+C \sum_{i=1}^{2} L^{n+1} P^{n+1} \\
& +C \sum_{i=1}^{2} v_{i}^{-3}\left(1+\left\|u_{2}^{n}\right\|_{I}^{4}+\left\|\left[\mathbf{u}\left(t^{n+1}\right)\right]\right\|_{I}^{4}\right)\left\|e_{i}^{n+1}\right\|_{0}^{2}+C \sum_{i=1}^{2}\left\|e_{i}^{n}\right\|_{0}^{2}\left\|\nabla u_{i}\left(t^{n+1}\right)\right\|_{0}^{2^{d}} \\
& +C \sum_{i=1}^{2} v_{i}^{-3} M^{n+1}\left(\left\|e_{i}^{n}\right\|_{0}^{2}+\left\|e_{i}^{n-1}\right\|_{0}^{2}\right) .
\end{aligned}
$$

Noting the bounds of $\left\|\nabla u_{i}\left(t^{n+1}\right)\right\|_{0}, \sum_{n=0}^{m-1}\left\|\nabla u_{i}^{n+1}\right\|_{0}$, and $\left\|\mathbf{u}_{t t}\right\|_{0}$ yields

$$
\begin{aligned}
& \left\|E_{i}^{n+1}-E_{i}^{n}\right\|_{-1}^{2}+\tau\left\|R H S_{i}^{n+1}\right\|_{-1}^{2} \\
& \quad \leq C\left(\tau^{2}+h^{2}\right)\left(\left\|\mathbf{u}_{t t}\right\|_{L^{2}\left(\left[t_{n-1}, t_{n+1}\right], L^{2}\left(\Omega_{i}\right)\right)}^{2}\right. \\
& \left.\quad+\|\mathbf{u}\|_{L^{2}\left(\left[t_{n-1}, t_{n+1}\right], H^{2}\left(\Omega_{i}\right)\right)}+\|p\|_{L^{2}\left(\left[t_{n-1}, t_{n+1}\right], H^{1}\left(\Omega_{i}\right)\right)}\right), \\
& L^{n+1} P^{n+1} \\
& \quad \leq C\left(\tau^{2}+h^{2 r}\right)\left(\left\|\mathbf{u}_{t}\right\|_{L^{2}\left(\left[t_{n-1}, t_{n+1}\right], L^{2}\left(\Omega_{i}\right)\right)}^{2}+\|\mathbf{u}\|_{L^{2}\left(\left[t_{n-1}, t_{n+1}\right], H^{2}\left(\Omega_{i}\right)\right)}^{2}\right) .
\end{aligned}
$$

Summing over $n=1,2, \ldots, m-1$, multiplying by $2 \tau$, using the classical estimates, and rewritting the last term of the right-hand side of the above inequality as

$$
\begin{aligned}
& \sum_{n=1}^{m-1}\left(\sum_{i=1}^{2} v_{i}^{-3} M^{n+1}\left(\left\|e_{i}^{n}\right\|_{0}^{2}+\left\|e_{i}^{n-1}\right\|_{0}^{2}\right)\right) \\
& \quad \leq C \sum_{i=1}^{2} v_{i}^{-3}\left(M^{2}+M^{3}\right)\left(\left\|e_{i}^{1}\right\|_{0}^{2}+\left\|e_{i}^{0}\right\|_{0}^{2}\right)+C \sum_{n=1}^{m-1} v_{i}^{-3}\left(M^{n+1}+M^{n+2}\right)\left\|\mathbf{e}^{n}\right\|_{0}^{2}
\end{aligned}
$$

we obtain

$$
\begin{aligned}
\left\|\mathbf{e}^{m}\right\|_{0}^{2} & +\sum_{n=1}^{m-1}\left\|\mathbf{e}^{n+1}-\mathbf{e}^{n}\right\|_{0}^{2}+\tau \sum_{n=1}^{m-1} \sum_{i=1}^{2} \frac{v_{i}}{2}\left\|\nabla e_{i}^{n+1}\right\|_{0}^{2} \\
& +\tau \sum_{i=1}^{2} \frac{v_{i}}{16}\left(\left\|\nabla e_{i}^{m-1}\right\|_{0}^{2}+4\left\|\nabla e_{i}^{m}\right\|_{0}^{2}\right) \\
\leq & \tau \sum_{i=1}^{2} \frac{v_{i}}{16}\left(\left\|\nabla e_{i}^{0}\right\|_{0}^{2}+4\left\|\nabla e_{i}^{1}\right\|_{0}^{2}\right) \\
& +\left(1+C \tau v_{i}^{-3}\left(M^{2}+M^{3}\right)\right)\left(\left\|\mathbf{e}^{0}\right\|_{0}^{2}+\left\|\mathbf{e}^{1}\right\|_{0}^{2}\right) \\
& +C\left(\tau^{2}+h^{2 r}\right)+C \tau \sum_{n=1}^{m-1} D^{n+1}\left\|\mathbf{e}^{n+1}\right\|_{0}^{2},
\end{aligned}
$$

where $D^{n+1}$ is defined by

$$
\begin{aligned}
D^{n+1}= & \left(v_{i}^{-3}\left(1+M^{n}+M^{n+1}+\left\|u_{2}^{n}\right\|_{I}^{4}+\left\|\left[\mathbf{u}\left(t^{n+1}\right)\right]\right\|_{I}^{4}\right)\right. \\
& \left.+\left\|\nabla \mathbf{u}\left(t^{n+1}\right)\right\|_{0}^{2^{d}}\right), \quad d=2,3
\end{aligned}
$$


and $C$ is dependent of the data $\left(\Omega_{i}, v_{i}, f_{i}\right)$. Setting

$$
a^{j+1}=\left\|e^{j+1}\right\|_{0}^{2}, \quad b^{j+1}=\left\|e^{j+1}-e^{j}\right\|_{0}^{2}, \quad c^{j+1}=\sum_{i=1}^{2}\left\|\nabla e_{i}^{j+1}\right\|_{0}^{2},
$$

and using Gronwall's inequality in Lemma 4.1, (28) and (42) yields the desired result.

\section{Numerical results}

In this section, we assess numerical performance of the stabilized methods for the presented model. It will be checked by a known analytical solution problem. The main goal of the experiment is to verify convergence rates of the scheme (16)-(17). Here, we denote errors by

$$
\begin{aligned}
& \operatorname{Err}\left(u_{i}\right)=\left(\tau \sum_{n=0}^{m}\left\|\nabla\left(u_{i}\left(t^{n+1}\right)-u_{i}^{n+1}\right)\right\|_{0, \Omega_{i}}^{2}\right)^{1 / 2}, \\
& \operatorname{Err}\left(p_{i}\right)=\left(\tau \sum_{n=0}^{m}\left\|p_{i}\left(t^{n+1}\right)-p_{i}^{n+1}\right\|_{0, \Omega_{i}}^{2}\right)^{1 / 2},
\end{aligned}
$$

where $i=1,2$. All numerical computations are implemented by open source software Freefem [19].

Example 1 The computations of the experiment are carried out in the domains $\Omega_{1}=$ $(0,1) \times(0,1)$ and $\Omega_{2}=(0,1) \times(-1,0)$. The prescribed exact solutions are given [13, 37] by

$$
\begin{aligned}
p_{1}(t, x, y)= & p_{2}(t, x, y)=\exp (-t) \cos (\pi x) \sin (\pi y), \\
u_{1,1}(t, x, y)= & -\alpha x^{2} \exp (-t)(x-1)^{2}(y-1), \\
u_{1,2}(t, x, y)= & \alpha x y \exp (-t)\left(6 x+y-3 x y+2 x^{2} y-4 x^{2}-2\right), \\
u_{2,1}(t, x, y)= & -\alpha x \exp (-t)(x-1)\left(y^{2} x(x-1)\left(\frac{\mu_{1}}{\mu_{2}}+1\right)\right. \\
& \left.-\frac{\mu_{1}^{1 / 2} y^{2} \exp (t / 2)}{(\alpha \kappa)^{1 / 2}}-x(x-1)+\frac{\mu_{1}^{1 / 2} \exp (t / 2)}{(\alpha \kappa)^{1 / 2}}+\frac{\mu_{1} x y(x-1)}{\mu_{2}}\right), \\
u_{2,2}(t, x, y)= & -\frac{\alpha y \exp (-t)(2 x-1)}{3 \mu_{2}(\alpha \kappa)^{1 / 2}}\left(6 \mu_{2} x^{2}(\alpha \kappa)^{1 / 2}-6 \mu_{2} x(\alpha \kappa)^{1 / 2}-3 \mu_{1}^{1 / 2} \mu_{2} \exp (t / 2)\right. \\
& -2 \mu_{1} x^{2} y^{2}(\alpha \kappa)^{1 / 2}-2 \mu_{2} x^{2} y^{2}(\alpha \kappa)^{1 / 2}+3 \mu_{1} x y(\alpha \kappa)^{1 / 2}+2 \mu_{1} x y^{2}(\alpha \kappa)^{1 / 2} \\
& \left.-3 \mu_{1} x^{2} y(\alpha \kappa)^{1 / 2}+2 \mu_{2} x y^{2}(\alpha \kappa)^{1 / 2}+\mu_{1}^{1 / 2} \mu_{2} y^{2} \exp (t / 2)\right),
\end{aligned}
$$

with an arbitrary positive constant $\alpha$. Here, $\left(u_{i}, p_{i}\right), i=1,2$ are the solutions of the original problem (1)-(6) and the right-hand sides $f=\left(f_{1}, f_{2}\right)$ can be obtained by (1). Moreover, $u_{2}=\left(u_{2,1}, u_{2,2}\right)$ satisfies the three interface conditions in [13].

Firstly, in the first example, we choose the same parameter values $\mu_{1}=0.5, \mu_{2}=0.05$, $\alpha=1$ and $\kappa=100$ as in [13]. The Euler scheme is used for the time discretization at $T=1$, 
Table 1 Errors for stabilized $P_{1}-P_{1}$ pair with $\tau=h$

\begin{tabular}{rllllllll}
\hline $1 / h$ & $\operatorname{Err}\left(u_{1}\right)$ & Rate & $\operatorname{Err}\left(u_{2}\right)$ & $\operatorname{Rate}$ & $\operatorname{Err}\left(p_{1}\right)$ & Rate & $\operatorname{Err}\left(p_{2}\right)$ & Rate \\
\hline 8 & $7.2271 \mathrm{E}-2$ & - & $2.9717 \mathrm{E}-1$ & - & $1.4967 \mathrm{E}-2$ & - & $1.1617 \mathrm{E}-2$ & - \\
32 & $1.7025 \mathrm{E}-2$ & 1.043 & $7.0463 \mathrm{E}-2$ & 1.038 & $1.8549 \mathrm{E}-3$ & 1.506 & $1.9019 \mathrm{E}-3$ & 1.305 \\
64 & $8.4129 \mathrm{E}-3$ & 1.017 & $3.4862 \mathrm{E}-2$ & 1.015 & $6.8180 \mathrm{E}-4$ & 1.444 & $7.2067 \mathrm{E}-4$ & 1.400 \\
\hline
\end{tabular}

Table 2 Errors for stabilized $P_{2}-P_{2}$ pair with $\tau=h^{2}$

\begin{tabular}{rllllllll}
\hline $1 / h$ & $\operatorname{Err}\left(u_{1}\right)$ & $\operatorname{Rate}$ & $\operatorname{Err}\left(u_{2}\right)$ & $\operatorname{Rate}$ & $\operatorname{Err}\left(p_{1}\right)$ & $\operatorname{Rate}$ & $\operatorname{Err}\left(p_{2}\right)$ & $\operatorname{Rate}$ \\
\hline 4 & $2.6926 \mathrm{E}-2$ & - & $1.7999 \mathrm{E}-1$ & - & $1.7270 \mathrm{E}-2$ & - & $1.6852 \mathrm{E}-2$ & - \\
8 & $5.7926 \mathrm{E}-3$ & 2.215 & $3.4207 \mathrm{E}-2$ & 2.396 & $4.2766 \mathrm{E}-3$ & 2.014 & $4.2613 \mathrm{E}-3$ & 1.984 \\
16 & $1.3445 \mathrm{E}-3$ & 2.107 & $6.9341 \mathrm{E}-3$ & 2.303 & $1.0623 \mathrm{E}-3$ & 2.009 & $1.1131 \mathrm{E}-3$ & 1.914 \\
\hline
\end{tabular}

Table 3 Errors for the different small viscosities based on stabilized $P_{1}-P_{1}$ pair

\begin{tabular}{llll}
\hline & $\mu_{1}=1.0 \mathrm{E}-4$ & $\mu_{1}=1.0 \mathrm{E}-4$ & $\mu_{1}=1.0 \mathrm{E}-5$ \\
& $\mu_{2}=1.0 \mathrm{E}-4$ & $\mu_{2}=1.0 \mathrm{E}-5$ & $\mu_{2}=1.0 \mathrm{E}-5$ \\
\hline $\operatorname{Err}\left(u_{1}\right)$ & $1.3843 \mathrm{E}-2$ & $1.3838 \mathrm{E}-2$ & $1.3864 \mathrm{E}-2$ \\
$\operatorname{Err}\left(u_{2}\right)$ & $2.0461 \mathrm{E}-2$ & $5.3043 \mathrm{E}-2$ & $2.0475 \mathrm{E}-2$ \\
$\operatorname{Err}\left(p_{1}\right)$ & $1.9068 \mathrm{E}-3$ & $1.9070 \mathrm{E}-3$ & $1.9063 \mathrm{E}-3$ \\
$\operatorname{Err}\left(p_{2}\right)$ & $9.7819 \mathrm{E}-4$ & $2.6972 \mathrm{E}-3$ & $9.7897 \mathrm{E}-4$ \\
\hline
\end{tabular}

Table 4 Errors for the different small viscosities based on stabilized $P_{2}-P_{2}$ pair

\begin{tabular}{llll}
\hline & $\mu_{1}=1.0 \mathrm{E}-4$ & $\mu_{1}=1.0 \mathrm{E}-4$ & $\mu_{1}=1.0 \mathrm{E}-5$ \\
& $\mu_{2}=1.0 \mathrm{E}-4$ & $\mu_{2}=1.0 \mathrm{E}-5$ & $\mu_{2}=1.0 \mathrm{E}-5$ \\
\hline $\operatorname{Err}\left(u_{1}\right)$ & $4.9393 \mathrm{E}-2$ & $4.9283 \mathrm{E}-2$ & $5.4135 \mathrm{E}-2$ \\
$\operatorname{Err}\left(u_{2}\right)$ & $4.9196 \mathrm{E}-2$ & $5.3924 \mathrm{E}-2$ & $5.3904 \mathrm{E}-2$ \\
$\operatorname{Err}\left(p_{1}\right)$ & $3.0189 \mathrm{E}-4$ & $3.0190 \mathrm{E}-4$ & $3.0190 \mathrm{E}-4$ \\
$\operatorname{Err}\left(p_{2}\right)$ & $3.1022 \mathrm{E}-4$ & $7.7659 \mathrm{E}-4$ & $3.1042 \mathrm{E}-4$ \\
\hline
\end{tabular}

with the time step $\tau=h$. Three values of space size $h=1 / 8,1 / 32,1 / 64$ are chosen. We display the convergence orders and errors of the presented method in Tables $1-2$ by $P_{r}-$ $P_{r}, r=1,2$. From Tables $1-2$, it can be easily seen that the method completely agree with the expected results in theory.

Secondly, we test the presented method with small viscosities. Here, we choose $\alpha=1$, $\kappa=100, h=1 / 20$ and the time step $\tau=0.005$. Then, we list the numerical errors with different small viscosities at $T=0.1$ in Tables 3-4. Obviously, the presented method can deal with these problems involving small viscosities.

Example 2 In this example, we test the presented method for a submarine mountain problem. This problem describes the fluid, which flows in a domain including the submarine mountain. In this case, the subdomain $\Omega_{2}$ is nonconvex. As is known, the viscosity of the fluid at submarine location is bigger than that at surface location. So we take $\mu_{1}=0.001$ and $\mu_{2}=0.01$ in this example.

Set $\Omega_{1}=[0,1] \times[0,0.1]$ and $\Omega_{2}=\left\{(x, y): \frac{7}{40}(1-(2 x-1) \sin (7 x-3.5)) \leq y \leq 0\right\}$. The initial conditions are chosen as follows:

$$
\begin{aligned}
& p_{1}(0, x, y)=p_{2}(0, x, y)=\cos (\pi x) \sin (\pi y), \\
& u_{1,1}(0, x, y)=x^{2}(1-x)^{2}(0.1-y),
\end{aligned}
$$



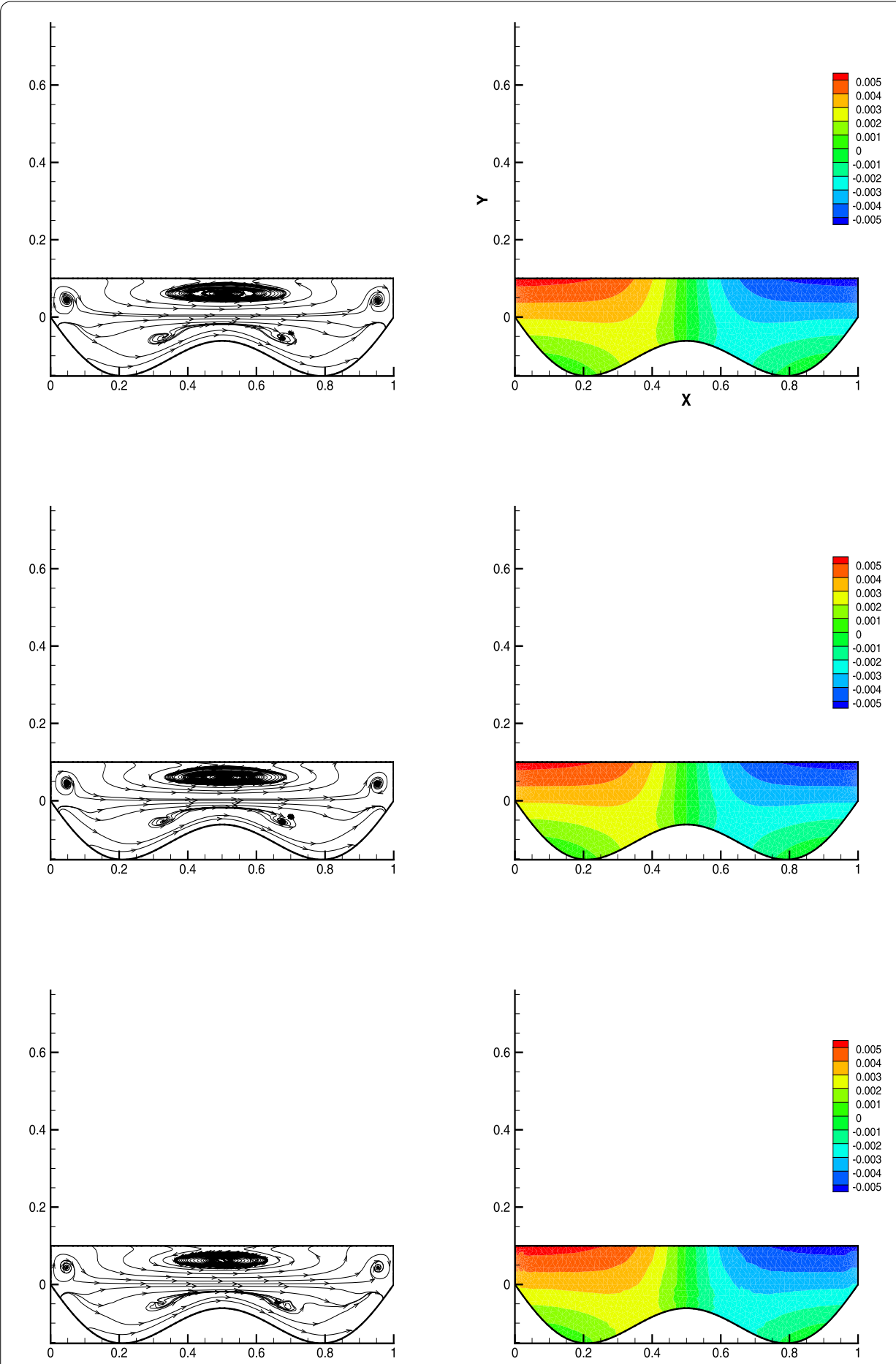

Figure 1 The numerical streamlines and isobars: the stabilized $P_{2}-P_{2}$ pair (the first line), the $P_{2}-P_{1}$ pair (the second line) and the stabilized $P_{1}-P_{1}$ pair (the third line)

$$
\begin{aligned}
& u_{1,2}(0, x, y)=x y\left(-0.2+y+0.6 x-3 x y-0.4 x^{2}+2 x^{2} y\right), \\
& u_{2,1}(0, x, y)=x^{2}(1-x)^{2}(0.1+y), \\
& u_{2,2}(0, x, y)=x y\left(-0.2-y+0.6 x+3 x y-0.4 x^{2}-2 x^{2} y\right) .
\end{aligned}
$$


We apply the presented method to get the numerical solution with $h=1 / 70$ and $\tau=1 / 40$. In Fig. 1, we present profiles for the numerical velocity and pressure with different methods at the final time $T=5$ and $\kappa=100$. From this figure, we can see that the stabilized methods are stable and the unphysical oscillations do not appear, and the numerical results of these stabilized methods completely agreement with those obtained by the classical $P_{2}-P_{1}$ pair [35]. Besides, we can find that the presence of the submarine mountain affects the fluid.

\section{Acknowledgements}

The authors express their sincere thanks to the anonymous reviewers for their valuable suggestions and corrections for improving the quality of this paper.

\section{Funding}

This work is supported by NSF of China (No. 11771259 and 11861067).

\section{Availability of data and materials}

Data sharing not applicable to this article as no datasets were generated or analyzed during the current study.

\section{Competing interests}

The authors declare that they have no competing interests.

\section{Authors' contributions}

All authors contributed equally to the writing of this paper. All authors read and approved the final manuscript.

\section{Author details}

${ }^{1}$ Department of Mathematics, School of Arts and Sciences, Shaanxi University of Science and Technology, Xian, P.R. China. ${ }^{2}$ College of Mathematics and System Sciences, Xinjiang University, Urumqi, China. ${ }^{3}$ School of Mathematics and Statistics, Xi'an Jiaotong University, Xi'an, P.R. China. ${ }^{4}$ Department of Chemical \& Petroleum Engineering, Schulich School of Engineering, University of Calgary, Calgary, Canada.

\section{Publisher's Note}

Springer Nature remains neutral with regard to jurisdictional claims in published maps and institutional affiliations.

Received: 27 December 2018 Accepted: 4 June 2019 Published online: 03 July 2019

\section{References}

1. Adams, R.A.: Sobolev Spaces. Academic Press, New York (1975)

2. Becker, R., Braack, M.: A finite element pressure gradient stabilization for the Stokes equations based on local projections. Calcolo 38, 173-199 (2001)

3. Bernardi, C., Chacón-Rebollo, T., Lewandowski, R., Murat, F.: Existence of a solution for a model of two coupled turbulent fluids. SIAM J. Numer. Anal. 40, 2368-2394 (2002)

4. Bresch, D., Koko, J.: Operator-splitting and Lagrange multiplier domain decomposition methods for numerical simulation of two coupled Navier-Stokes fluids. Int. J. Appl. Math. Comput. Sci. 16, 419-429 (2006)

5. Brezzi, F., Fortin, M.: Mixed and Hybrid Finite Element Methods. Springer, New York (1991)

6. Brezzi, F., Fortin, M.: A minimal stabilisation procedure for mixed finite element methods. Numer. Math. 89, 457-491 (2001)

7. Brezzi, F., Pitkäranta, J.: On the stabilization of finite element approximations of the Stokes equations. In: Proceedings on the Efficient Solutions of Elliptic Systems, Kiel, 1984. Notes on Numerical Fluid Mechanics, vol. 10, p. 11. Vieweg, Braunschweig (1984)

8. Burman, E.: Pressure projection stabilizations for Galerkin approximations of Stokes' and Darcy's problem. Numer. Methods Partial Differ. Equ. 24, 127-143 (2008)

9. Chen, Z.: Finite Element Methods and Their Applications. Springer, Heidelberg (2005)

10. Chen, Z., Wang, Z., Zhu, L., Li, J.: Analysis of the pressure projection stabilization method for the Darcy and coupled Darcy-Stokes flows. Comput. Geosci. 17, 1079-1091 (2013)

11. Ciarlet, P.G. The Finite Element Method for Elliptic Problems. North-Holland, Amsterdam (1978)

12. Connors, J., Howell, J., Layton, W.: Decoupled time stepping methods for fluid-fluid interaction. SIAM J. Numer. Anal. 50, 1297-1319 (2012)

13. Connors, J.M., Howell, J.S.: A fluid-fluid interaction method using decoupled subproblems and differing time steps. Numer. Methods Partial Differ. Equ. 28, 1283-1308 (2012)

14. Dohrmann, C.R., Bochev, P.B.: A stabilized finite element method for the Stokes problem based on polynomia pressure projections. Int. J. Numer. Methods Fluids 46, 183-201 (2004)

15. Girault, V., Raviart, P.A.: Finite Element Method for Navier-Stokes Equations: Theory and Algorithms. Springer, Berlin (1987)

16. He, Y., Li, J.: Convergence of three iterative methods based on the finite element discretization for the stationary Navier-Stokes equations. Comput. Methods Appl. Mech. Eng. 198, 1351-1359 (2009)

17. He, Y., Li, J.: Numerical comparisons of time-space iterative method and spatial iterative methods for the stationary Navier-Stokes equations. J. Comput. Phys. 231, 6790-6800 (2012) 
18. He, Y., Lin, Y., Sun, W.: Stabilized finite element method for the non-stationary Navier-Stokes problem. Discrete Contin. Dyn. Syst., Ser. B 6, 41-68 (2006)

19. Hecht, F., LeHyaric, A., Pironneau, O.: Freefem++ version 3.23 (2013) http://www.freefem.org/ff++

20. Heywood, J.G., Rannacher, R.: Finite-element approximations of the nonstationary Navier-Stokes problem. Part I: regularity of solutions and second-order spatial discretization. SIAM J. Numer. Anal. 19, 275-311 (1982)

21. Heywood, J.G., Rannacher, R.: Finite-element approximation of the nonstationary Navier-Stokes problem part IV: error analysis for second-order time discretization. SIAM J. Numer. Anal. 27, 353-384 (1990)

22. Huang, P., Feng, X., He, Y.: A quadratic equal-order stabilized finite element method for the conduction-convection equations. Comput. Fluids 86, 169-176 (2013)

23. Layton, W.: Model reduction by constraints, discretization of flow problems and an induced pressure stabilization. Numer. Linear Algebra Appl. 12, 547-562 (2005)

24. Layton, W.: Introduction to the Numerical Analysis of Incompressible Viscous Flows. Comput. Sci. Eng., vol. 6. SIAM, Philadelphia (2008)

25. Layton, W., Tran, H., Trenchea, C.: Analysis of long time stability and errors of two partitioned methods for uncoupling evolutionary groundwater-surface water flows. SIAM J. Numer. Anal. 51, 248-272 (2013)

26. Li, J., Chen, Z.: A new stabilized finite volume method for the stationary Stokes equations. Adv. Comput. Math. 30, 141-152 (2009)

27. $L i, J .$, Chen, Z:: Optimal $L^{2}, H^{1}$ and $L^{\infty}$ analysis of finite volume methods for the stationary Navier-Stokes equations with large data. Numer. Math. 126, 75-101 (2014)

28. Li, J., Chen, Z., He, Y.: A stabilized multi-level method for non-singular finite volume solutions of the stationary 3D Navier-Stokes equations. Numer. Math. 122, 279-304 (2012)

29. Li, J., He, Y.: A new stabilized finite element method based on two local Gauss integration for the Stokes equations. J. Comput. Appl. Math. 214, 58-65 (2008)

30. Li, J., He, Y., Chen, Z.: A new stabilized finite element method for the transient Navier-Stokes equations. Comput. Methods Appl. Mech. Eng. 197, 22-35 (2007)

31. Lions, J.L., Temam, R., Wang, S.: Models for the coupled atmosphere and ocean (CAO I). Comput. Mech. Adv. 1, 1-54 (1993)

32. Lions, J.L., Temam, R., Wang, S.: Numerical analysis of the coupled atmosphere-ocean models (CAO II). Comput. Mech. Adv. 1, 55-119 (1993)

33. Nasserdine, K., Silvester, D.: Analysis of locally stabilized mixed finite element methods for the Stokes problem. Math Compet. 58, 1-10 (1992)

34. Silvester, D.: Optimal low order finite element methods for incompressible flow. Comput. Methods Appl. Mech. Eng. $111,357-368(1994)$

35. Taylor, C., Hood, P.: A numerical solution of the Navier-Stokes equations using the finite element technique. Comput. Fluids 1, 73-100 (1973)

36. Temam, R.: Navier-Stokes Equations, Theory and Numerical Analysis, 3rd edn. North-Holland, Amsterdam (1984)

37. Zhang, H., Hou, Y., Shan, L.: Stability and convergence analysis of decoupled algorithm for fluid-fluid interaction. SIAM J. Numer. Anal. 54, 2833-2867 (2016)

38. Zheng, H., Shan, L., Hou, Y.: A quadratic equal-order stabilized method for Stokes problem based on two local Gauss integrations. Numer. Methods Partial Differ. Equ. 26, 1180-1190 (2010)

\section{Submit your manuscript to a SpringerOpen ${ }^{\circ}$ journal and benefit from:}

- Convenient online submission

- Rigorous peer review

- Open access: articles freely available online

- High visibility within the field

- Retaining the copyright to your article

Submit your next manuscript at $\boldsymbol{s p r i n g e r o p e n . c o m ~}$ 